\title{
Anomaly inflow mechanism using Wilson line
}

\author{
Wei Liao $^{1}$ \\ 1 TRIUMF, 4004 Wesbrook Mall, Vancouver, BC V6T 2A3, Canada
}

\begin{abstract}
It is shown that the anomaly inflow mechanism can be implemented using Wilson line in odd dimensional gauge theories. An action of Wess-Zumino-Witten (WZW) type can be constructed using Wilson line. The action is understood in the odd dimensional bulk space-time rather than in the even dimensional boundary. This action is not gauge invariant. It gives anomalous gauge variations of the consistent form on boundary space-times. So it can be used to cancel the quantum anomalies localized on boundary space-times. This offers a new way to cancel the gauge anomaly and construct anomaly-free gauge theory in odd dimensional space-time.
\end{abstract}

When embedding an even $(2 n)$ dimensional spacetime $\mathcal{M}$ into an odd $(2 n+1)$ dimensional space-time $\Sigma$ as a boundary, $\mathcal{M}=\partial \Sigma$, massless chiral fermion can be localized on the even dimensional space-time $\mathcal{M}$. Chiral fermion on $\mathcal{M}$ when coupled to gauge field can induce gauge anomaly via radiative corrections and can give potential problem to the gauge invariance. The gauge invariance can be maintained and the quantum anomaly on boundary can be canceled by anomalous gauge variation of a classical action in the odd dimensional bulk space-time $\Sigma$. This is the anomaly inflow mechanism. The mechanism states that the anomalous gauge variation given by the bulk action flows into boundary and cancels the anomalous gauge variation localized on the boundary. Using this mechanism one can construct consistent models in odd dimensional space-times with localized chiral degrees of freedom on even dimensional boundaries. The conventional way to achieve the cancellation is to use the Chern-Simons action in the bulk [1].

An important application of this mechanism is in condensed matter physics. It is well known that one can understand the quantum Hall effect using this mechansim 2, 3]. The mechanism has been generalized to more complicated theories, e.g. the theory of gravitation and the theory with gauge field of more than one indices. They have been applied in string theory and the building of extra dimensional models [3]. In this article we show that there is a new way to implement the anomaly inflow mechanism in odd dimensional gauge theories, hence a new way to build anomaly-free gauge theories in odd dimensional spacetimes. This is achieved using an action of WZW type constructed using Wilson line. In the following we give a simple example to illustrate the point of this article. We first introduce notations. Then we describe the anomaly induced by chiral fermion localized on boundary. The crucial bulk action to cancel anomaly is then introduced and its property is discussed.

Consider an example on $\Sigma_{3}=\mathcal{M}_{2} \times[0, \pi R]$ with coordinate $x^{M}=\left(x^{\mu}, x^{2}=y\right)(\mu=0,1) . \mathcal{M}_{2}$ is the two dimensional Minkowski space-time. $\Sigma_{3}$ has two boundaries of type $\mathcal{M}_{2}$ at $y=0$ and $y=\pi R$ separately. The gauge field $A_{M}=\sum_{a} T^{a} A_{M}^{a}$ of the gauge group $G$ propagates in the bulk. $T^{a}$ is the generator of the group $G$. We define $A_{M}$ having dimension [M]. Gauge coupling is absorbed into the gauge field. We denote the boundary branes at $y=0$ and $y=\pi R$ as brane $\mathrm{L}$ and brane $\mathrm{R}$ respectively. We introduce gauge fields on the boundary branes, $A_{L \mu}$ and $A_{R \mu}$, which are obtained when reducing $A_{\mu}$ to the boundaries:

$$
\begin{aligned}
& A_{L \mu}\left(x^{\mu}\right)=A_{\mu}\left(x^{\mu}, y=0\right), \\
& A_{R \mu}\left(x^{\mu}\right)=A_{\mu}\left(x^{\mu}, y=\pi R\right) .
\end{aligned}
$$

$A_{L, R \mu}$ live on two dimensional space-times and have no indices along $y$ direction. Massless chiral fermions $\psi_{L}$ and $\psi_{R}$ are localized on brane $\mathrm{L}$ and brane $\mathrm{R}$ respectively. They are minimally coupled to $A_{L}$ and $A_{R}$ separately, see Fig. 1.

We study the property of gauge theory under infinitesimal gauge transformation $U$ which approaches unity at infinity:

$$
U\left(x^{\mu}, y\right)=e^{i \epsilon}, \quad \epsilon\left(x^{\mu}, y\right)=\sum_{a} T^{a} \epsilon^{a}\left(x^{\mu}, y\right) .
$$

We have

$$
\delta A_{M}=\partial_{M} \epsilon+i\left[\epsilon, A_{M}\right] .
$$

$A_{L, R}$ transform as

$$
\delta A_{L, R \mu}=\partial_{\mu} \epsilon_{L, R}+i\left[\epsilon_{L, R}, A_{L, R \mu}\right],
$$

where $\epsilon_{L, R}$ are

$$
\epsilon_{L}\left(x^{\mu}\right)=\epsilon\left(x^{\mu}, y=0\right), \quad \epsilon_{R}\left(x^{\mu}\right)=\epsilon\left(x^{\mu}, y=\pi R\right) .(6)
$$

The 2D chiral gauge theory is anomalous [4, 5], that is the classical gauge symmetry is broken at the 
quantum level. The anomaly can be stated that the Noether current derived from the classical Lagrangian is not conserved when including radiative corrections of chiral fermions [4, 5]. As an alternative it can be stated that the quantum action is not invariant under the gauge transformation of the classical Lagrangian [3]. In this $3 \mathrm{D}$ example it means the quantum actions localized on two boundaries $\mathrm{L}$ and $\mathrm{R}$ are not invariant under the gauge transformation given in (5).

We denote $\Gamma_{L}$ and $\Gamma_{R}$ as the quantum actions ${ }^{1}$

$$
\Gamma_{L}=\left.\Gamma_{e f f}\right|_{y=0}, \quad \Gamma_{R}=\left.\Gamma_{e f f}\right|_{y=\pi R} .
$$

Under the gauge transformation given in (5) we have [3]

$$
\begin{array}{r}
\delta \Gamma_{L}=-\frac{1}{4 \pi} \int_{\mathcal{M}_{2}} d^{2} x \varepsilon^{\mu \nu} \operatorname{Tr}\left[\epsilon_{L} \partial_{\mu} A_{L \nu}\right], \\
\delta \Gamma_{R}=\frac{1}{4 \pi} \int_{\mathcal{M}_{2}} d^{2} x \varepsilon^{\mu \nu} \operatorname{Tr}\left[\epsilon_{R} \partial_{\mu} A_{R \nu}\right],
\end{array}
$$

where $\varepsilon^{01}=1$. (8) and (9) are anomalous gauge variations on boundary space-times and are of the form of consistent anomaly in 2D space-time [5]. \pm signs arise from different chiralities in $2 \mathrm{D}$ theory $[3$.

In a consistent gauge theory anomalies in (8) and (9) have to be canceled by contribution of other sector of the theory. The cancellation of anomaly can be achieved when an action in 3D space-time gives anomalies flowing into the boundary space-times. The total anomaly can be arranged to cancel. This is conventionally achieved using the Chern-Simons action [3]. We point out that we can achieve anomaly cancellation using another action in the bulk space-time. This bulk action is constructed using Wilson line and is of WZW type. Under the gauge transformation (5) it gives

$$
\begin{aligned}
\delta \Gamma_{W Z W} & =\frac{1}{4 \pi} \int_{\mathcal{M}_{2}} \varepsilon^{\mu \nu} \operatorname{Tr}\left[\epsilon_{L} \partial_{\mu} A_{L \nu}\right] \\
& -\frac{1}{4 \pi} \int_{\mathcal{M}_{2}} \varepsilon^{\mu \nu} \operatorname{Tr}\left[\epsilon_{R} \partial_{\mu} A_{R \nu}\right] .
\end{aligned}
$$

It gives the consistent form of anomaly on two boundaries. It is clear that the gauge invariance is maintained in the action

$$
\Gamma=\Gamma_{W Z W}+\Gamma_{L}+\Gamma_{R}
$$

\footnotetext{
1 To avoid complication of renormalization, one needs to limit
} the discussion to quantum action of one-loop level.

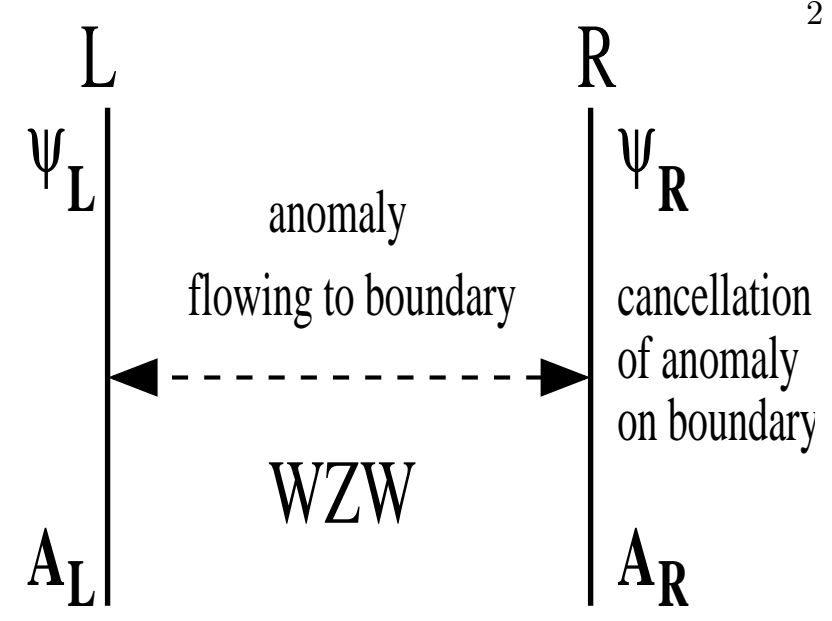

FIG. 1: Anomaly inflow mechanism implemented by WZW action. Gauge fields on $\mathrm{L}$ and $\mathrm{R}$ branes are induced by gauge field in the bulk: $A_{L \mu}\left(x^{\mu}\right)=A_{\mu}\left(x^{\mu}, y=0\right)$ and $A_{R \mu}\left(x^{\mu}\right)=A_{\mu}\left(x^{\mu}, y=\pi R\right)$.

The construction of the theory is shown in Fig. 1. We emphasize that this is a new way to achieve anomaly cancellation in 3D gauge theories. In the following we elaborate on this WZW type action in 3D space-time.

Note that the original WZW action is constructed in non-linear sigma model and is an action in $4 \mathrm{D}$ spacetime [6, 7, 8, 9, 10, 11]. Extension of this action to even dimensional space-time exists in the literature [12]. $\Gamma_{W Z W}$ used in this article should be understood living in three (odd) dimensional space-time, hence is a further extension of the original WZW action. This extension is achieved using the Wilson line along the third dimension [13, 14, 15]. We introduce Wilson line

$$
W_{y}\left(x^{\mu}, y\right)=\mathcal{P} e^{i \int_{0}^{y} d y^{\prime} A_{2}\left(x^{\mu}, y^{\prime}\right)},
$$

and

$$
W\left(x^{\mu}\right)=\mathcal{P} e^{i \int_{0}^{\pi R} d y^{\prime} A_{2}\left(x^{\mu}, y^{\prime}\right)}
$$

$\mathcal{P}$ is the path-ordering operator. $A_{2}$ is the gauge field along the third dimension. Since $W_{y}\left(x^{\mu}, y=0\right)=1$ the configuration of $W_{y}$ is the mapping of $\widetilde{\Sigma}_{3}$ to the space of the gauge group $G . \widetilde{\Sigma}_{3}$ is $\Sigma_{3}$ with the $2 \mathrm{D}$ boundary at $y=0$ shrinking to a point. So $\widetilde{\Sigma}_{3}$ has a single boundary: $\partial \widetilde{\Sigma}_{3}=\mathcal{M}_{2}$.

$W=W_{y}(y=\pi R)$ transforms as a bifundamental of gauge transformations on two boundary space-times:

$$
W^{\prime}\left(x^{\mu}\right)=U\left(x^{\mu}, y=0\right) W\left(x^{\mu}\right) U^{-1}\left(x^{\mu}, y=\pi R\right)(.14)
$$


$W$ is a link field which can mix the degrees of the freedom on two boundaries. Using it we can write an action [13]

$$
\begin{aligned}
\Gamma_{W Z W} & =-\frac{1}{12 \pi} \int_{\Sigma_{3}} d^{3} x \varepsilon^{R S T} \operatorname{Tr}\left[\left(\partial_{R} W_{y}\right) W_{y}^{-1}\right. \\
& \left.\times\left(\partial_{S} W_{y}\right) W_{y}^{-1}\left(\partial_{T} W_{y}\right) W_{y}^{-1}\right] \\
& -\frac{i}{4 \pi} \int_{\mathcal{M}_{2}} d^{2} x \varepsilon^{\mu \nu} \operatorname{Tr}\left[A_{L \mu} W_{L \nu}\right. \\
& \left.+A_{R \mu} W_{R \nu}-i A_{R \mu} W^{-1} A_{L \nu} W\right]
\end{aligned}
$$

where $^{2} R, S, T$ run over $0,1,2, \varepsilon^{012}=1$ and

$$
W_{L \mu}=\left(\partial_{\mu} W\right) W^{-1}, \quad W_{R \mu}=W^{-1}\left(\partial_{\mu} W\right)
$$

The action written in (15) is formally of WZW type and is similar to the WZW action in 2D space-time. This action has to be interpreted as living in 3D spacetime, quite different from the interpretation of the usual WZW action living in even dimensional spacetime [6]. One can understand this interpretation by noting that this action gives non-local interaction for gauge fields $A_{L}$ and $A_{R}$ on different boundary branes. That is it gives interaction for degrees of freedom at space-like distance. ${ }^{3}$ In particular, the last term in (15) can not be understood as living in any one of the two boundary branes. We emphasize that this action can not be understood as localized on any one of the boundary branes.

Gauge transformation property of (15) can be easily computed. Note that under the infinitesimal transformation (3) and (41) we have

$$
\delta W_{y}\left(x^{\mu}, y\right)=i \epsilon_{L} W_{y}\left(x^{\mu}, y\right)-i W_{y}\left(x^{\mu}, y\right) \epsilon_{y}
$$

where

$$
\epsilon_{y}\left(x^{\mu}\right)=\epsilon\left(x^{\mu}, y\right)
$$

Using (5) and (17) we can easily get the transformation property of $\Gamma_{W Z W}$. Dropping the surface integration at infinity Eq. (10) is obtained.

The above construction in $3 \mathrm{D}$ can be generalized to $5 \mathrm{D}$ space-times with boundaries. One can also take

\footnotetext{
2 There is a difference on sign compared to the action written in Ref. [13].

3 This non-locality along the compact space-like dimension may not affect the $2 \mathrm{D}$ locality. A proof for this statement is needed.
}

the picture shown in Fig. 1] $\psi_{L}$ and $\psi_{R}$ are then understood as localized on 4D boundaries. Similarly we have quantum actions $\Gamma_{L}$ and $\Gamma_{R}$ on two boundary branes which are obtained including radiative corrections of $\psi_{L}$ and $\psi_{R}$ separately. Gauge anomaly in 4D space-time states that $\Gamma_{L}$ and $\Gamma_{R}$ are not gauge invariant [3, 13]. For an infinitesimal gauge transformation, they give

$$
\begin{aligned}
& \delta \Gamma_{L}=-\frac{1}{24 \pi^{2}} \int_{\mathcal{M}_{4}} d^{4} x \omega_{4}^{1}\left(A_{L \mu}, \epsilon_{L}\right), \\
& \delta \Gamma_{R}=\frac{1}{24 \pi^{2}} \int_{\mathcal{M}_{4}} d^{4} x \omega_{4}^{1}\left(A_{R \mu}, \epsilon_{R}\right),
\end{aligned}
$$

where

$$
\omega_{4}^{1}\left(B_{\mu}, \varepsilon\right)=\varepsilon^{\mu \nu \rho \sigma} \operatorname{Tr}\left[\varepsilon \partial_{\mu}\left(B_{\nu} \partial_{\rho} B_{\sigma}-\frac{i}{2} B_{\nu} B_{\rho} B_{\sigma} \backslash 21\right)\right.
$$

$\mu, \nu, \rho, \sigma$ run over $0,1,2,3$ and $\varepsilon^{0123}=1$. (19) and (20) are of the form of consistent anomaly in $4 \mathrm{D}$ spacetime [5].

A 5D WZW type action has been constructed using Wilson line in Ref. [13, 15]. It gives anomalous gauge variations flowing into the boundary branes. Readers are referred to Ref. [13] for explicit formula (an extra minus sign is needed to get the gauge variation (22) in the following). The action constructed is similar to the 4D WZW action [6, 7, 8, 9, 10, 11]. However it has to be understood in the $5 \mathrm{D}$ bulk rather than localized on any one of the boundary space-times. This action gives non-local interaction for gauge fields $A_{L}$ and $A_{R}$ of two boundary space-times. Under the infinitesimal gauge transformation it gives

$$
\begin{aligned}
\delta \Gamma_{W Z W} & =\frac{1}{24 \pi^{2}} \int_{\mathcal{M}_{4}} d^{4} x \omega_{4}^{1}\left(A_{L \mu}, \epsilon_{L}\right) \\
& -\frac{1}{24 \pi^{2}} \int_{\mathcal{M}_{4}} d^{4} x \omega_{4}^{1}\left(A_{R \mu}, \epsilon_{R}\right)
\end{aligned}
$$

The gauge variation on boundaries given by this action is of the form of consistent anomaly. It is clear that anomalous gauge variations of $\Gamma_{L}$ and $\Gamma_{R}$ are canceled by the gauge variation of the bulk action. This implements the anomaly inflow mechanism using Wilson line in 5D space-time.

This is the story to implement the anomaly inflow mechanism using Wilson line in 3D and 5D gauge theories. Using this mechanism one can construct anomaly-free gauge theories in $3 \mathrm{D}$ or $5 \mathrm{D}$ space-time with massless chiral fermions localized on boundaries, as shown in Fig. 11. We emphasize that this new mechanism is quite different from the old one using 
the Chern-Simons action. Because of the non-local nature of $\Gamma_{W Z W}$ we do not expect to find a current along the compact space-like dimension. On the contrary, the old mechanism indeed has a current along the compact dimension. This current is derivable from the Chern-Simons term.

As a further comparison we note that the WZWtype term used in this article and the Chern-Simons term have different origins in effective theory. In a previous work [13] we have shown how this WZW type action can be completely derived when integrating out some chiral fermions on even dimensional boundaries. These chiral fermions are localized on boundary spacetimes and are coupled to Wilson line via non-local Yukawa-type interaction. Integrating out these chiral fermions we find the WZW type action $\Gamma_{W Z W}$ appearing in the effective action. On the other hand, it is known for a long time that the Chern-Simons term appears in effective theory when integrating out fermion in odd dimensional space-time [16]. It is clear that $\Gamma_{W Z W}$ used in this article is different from and independent of the Chern-Simons action. This opinion is different from the opinion taken in [14]. We emphasize that the mechanism shown in this article is a new mechanism, not the old mechanism rewritten in a new form.

In summary we have shown that there is a new type of anomaly inflow mechanism implemented by a WZW type action constructed using Wilson line. This action has to be understood as living in odd dimensional space-time rather than in even dimensional space-time. This point is also emphasized in Ref. [13]. The action constructed is not gauge invariant and it gives anomalous gauge variations of the consistent form on boundary space-times. These anomalous gauge variations cancel the anomalous gauge variations localized on boundaries. Using this mechanism the gauge invariance of the odd dimensional gauge theory can be achieved when massless chiral fermions are localized on boundary space-times.

We expect that the 3D version of this new mechanism is of potential interests to condensed matter physics. It may have application similar to the application of the old mechanism using Chern-Simons term in quantum Hall effects. However the experimental devices need to be different. This topic requires further research. We expect that this mechanism can be generalized to odd dimensional space-times of dimension larger than five. Action to implement the mechanism will be similar to the WZW action in even dimensional space-times of dimension larger than four [12]. Examples shown in this article are for gauge theory in flat space-time. We expect this mechanism can be generalized to curved background space-time. Using this mechanism we are able to construct new type of anomaly-free gauge theories in odd dimensional space-times. Extension of this mechanism to more complicated theories, e.g. the theory of gravity, requires further research.

Acknowledgment: the author wishes to thank C. S. Lam for helpful comments.
[1] C. G. Callan and J. A. Harvey, Nucl. Phys. B250(1985) 427.

[2] Xiao-Gan Wen, Quantum Field Theory of Many-body Systems, Oxford Press 2004.

[3] For a brief review see A. Bilal, hep-th/0409028.

[4] R. Jackiw, in Relativity, Groups and Topology II (Les Houches lecture 1983), eds. B. S. DeWitt and R. Stora, Elsevier 1984.

[5] W. A. Bardeen and B. Zumino, Nucl. Phys. B244(1984) 421.

[6] J. Wess and B. Zumino, Phys. Lett. B37(1971)95; S. Novikov, Sov. Math. Dokl. 24(1981)222; E. Witten, Nucl. Phys. B223(1983)422.

[7] K-C. Chou, H-Y. Kuo, K. Wu and X-C. Song, Phys. Lett. B134(1984)67.

[8] Ö. Kaymakcalan, S. Rajeev and J. Schecher, Phys. Rev. D30(1984)594.

[9] H. Kawai and S-H. H. Tye, Phys. Lett.
B140(1984)403.

[10] A. Manohar and G. Moore, Nucl. Phys. B243(1984)55.

[11] J. L. Mañes, Nucl. Phys. B250(1985)369.

[12] Y-S. Wu, Phys. Lett. B153(1985)70.

[13] W. Liao, Phys. Rev. D74(2006)065010 hep-th/0605016.

[14] C. T. Hill, Phys. Rev. D73(2006)126009 hep-th/0603060.

[15] Ealier attempts to obtain WZW type action using Wilson line include Ref. [14]; C. T. Hill, Phys. Rev. D73(2006)085001 hep-th/0601154; hep-th/0601155. C. T. Hill and C. K. Zachos, Phys. Rev. D71(2005)046002 hep-th/0411157. The action was considered in $4 \mathrm{D}$, different from the interpretation emphasized in Ref. 13] and this article.

[16] A. N. Redlich, Phys. Rev. Lett 52(1984)18. 